\title{
Pulsed-laser Doppler flowmetry provides basis for deep perfusion probing
}

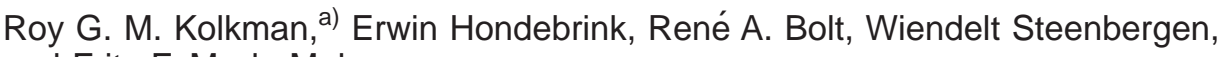 \\ and Frits F. M. de Mul \\ Department of Applied Physics, University of Twente, P.O. Box 217, NL-7500 AE Enschede, the Netherlands
}

(Received 14 March 2001; accepted for publication 14 August 2001)

\begin{abstract}
A setup for pulsed-laser Doppler flowmetry (LDF) measurements has been built and tested. Measurements were carried out comparing continuous-wave and pulsed LDF. With pulsed LDF a higher peak power can be injected into the tissue without exceeding the safety limits. This enables a much larger spacing between the locations of illumination and detection. Thus, the penetration depth, and thus the measurement volume, can be enlarged using the pulsed-LDF method. This method will allow, e.g., monitoring of the cerebral perfusion. () 2001 American Institute of
\end{abstract} Physics. [DOI: 10.1063/1.1409566]

\section{INTRODUCTION}

Laser Doppler flowmetry (LDF) provides a method for noninvasive measurement of the perfusion of tissue. The tissue is illuminated with a cw laser source and the backscattered light from the tissue is then detected and analyzed. Due to scattering of the light at moving red blood cells, this light gets a frequency shift, which causes the detector signal to fluctuate due to interference. These fluctuations contain information about the perfusion. Bonner and Nossal ${ }^{1}$ have shown that the first-order moment of the power spectral density of these signals is proportional to the root-mean-square velocities of the red blood cells:

$$
\int_{0}^{\infty} \omega S(\omega) d \omega \sim \sqrt{\left\langle\nu^{2}\right\rangle}
$$

Perfusion deep under the tissue surface can in principle be measured with laser Doppler flowmetry by placing the fibers for illumination and detection at a sufficient distance from each other. However, due to the strong attenuation of reflected light, laser powers would be needed which exceed the safety limits. Soelkner and co-workers ${ }^{2,3}$ have shown that it is possible to perform laser Doppler measurements up to a depth of $30 \mathrm{~mm}$ with $120 \mathrm{~mW} \mathrm{cw}$ light in a tissue-like head phantom. However, the high power needed prevented application on real tissue.

A way to increase the power without exceeding the safety limit is by pulsing the laser source and pulse sampling the Doppler signal. By using short pulses, a high peak power can be achieved with a low average power. For long-term monitoring of the perfusion the average power determines the maximum allowable power for skin illumination.

The developed pulsed-LDF method expressly makes use of the high peak power by sampling the peak signals, and thus enables noninvasive monitoring of deep perfusion. Other pulsed- ${ }^{4}$ or modulated- ${ }^{5}$ LDF methods do not make use of the higher peak power, but use the average power to determine the blood flow. Applications of the developed

a)Electronic mail: r.g.m.kolkman@tn.utwente.nl
pulsed-LDF method include noninvasive measurement of the brain perfusion as well as monitoring of the perfusion through organs (e.g., kidney).

To test the proposed LDF method a test setup has been constructed using a pulsed-laser diode.

\section{METHOD}

With tissue perfusion the typical frequency range of LDF is $0-20 \mathrm{kHz}$, corresponding with characteristic time scales up from $50 \mu$ s. These times are much larger than typical coherence times of the laser, which implies that each period of the Doppler signal will contain information from many coherence times. Then, it does not matter whether those coherence times were directly adjacent to each other (as is the case with $\mathrm{cw}$ ) or had intermittent time periods (as with pulsed LDF). Following this line, the pulsed-laser Doppler method has been designed.

The minimum pulse duration $t_{\text {pulse }}$ is limited by the maximum path-length difference $l_{\max }$, which can occur in the measurement volume: photons traveling along the shortest path length should have the opportunity to interfere at the detector with photons traveling along the longest path length. The minimum pulse duration can be estimated by

$$
t_{\text {pulse }}>\frac{l_{\max }}{c / n},
$$

with $n$ the refractive index of tissue and $c$ the speed of light in vacuum.

Assuming a maximum path-length difference of $50 \mathrm{~cm}$, which holds for nearly all practical cases, and $n=1.33$ gives a minimum pulse duration of $2.2 \mathrm{~ns}$. Furthermore, the coherence length of the laser has to be larger than the maximum path-length difference to make photons traveling along the shortest and longest path-length interfering at the detector: $l_{\text {coh }}>l_{\max }$.

As laser diodes are often used ${ }^{6-8}$ as a light source in LDF instruments, for this pulsed-LDF method also a laser diode has been used, which fulfills the mentioned criteria for the pulsed light source. 


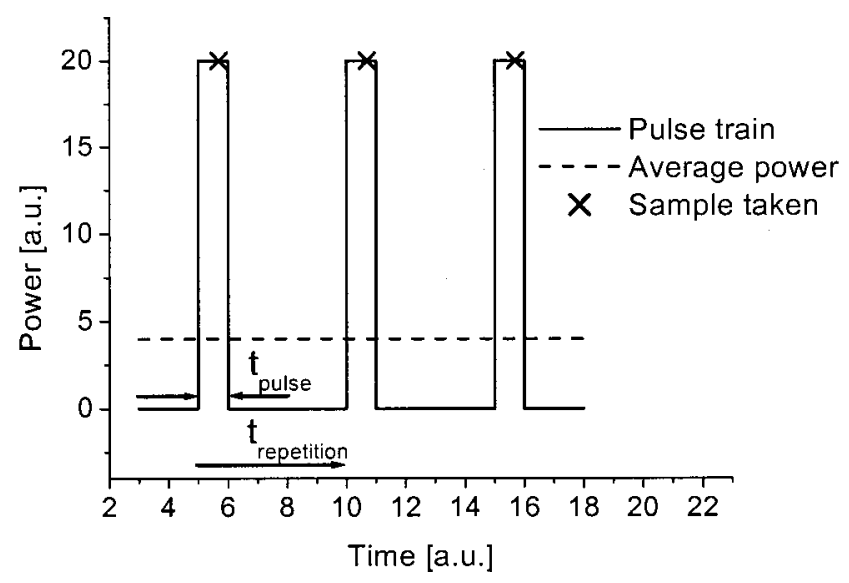

FIG. 1. Schematic representation of time scheme pulsed-LDF measurement.

The LDF time signals are collected by sampling with an analog-to-digital AD card. A sample is taken during the time the laser is "on." The laser is switched off for the time between two adjacent sample points (Fig. 1). The average power $P_{\text {avg }}$ during the LDF measurement is equal to the time ratio of the pulse width $t_{\text {pulse }}$ and the repetition time $t_{\text {repetition }}$ times the peak pulse power $P_{\text {peak }}$ :

$$
P_{\text {avg }}=\frac{t_{\text {pulse }}}{t_{\text {repetition }}} P_{\text {peak }} .
$$

The basic setup is shown in Fig. 2. A pulse generator (Hameg 8035 ) is used to modulate the laser diode driver (Thorlabs LDC 500) and provides the trigger for the AD card (National Instruments AT-MIO-16XE-50). The light pulses of the single-mode laser diode (Hitachi HL6501MG) are transmitted via an optical fiber (step index, NA $=0.23$ and core diameter $400 \mu \mathrm{m}$ ) to the sample. The backscattered and Doppler shifted light is transmitted through the same type of fiber to a photodiode (BPW 34). In this study the pulse generator delivers pulses of $16 \mu \mathrm{s}$ with a repetition rate of $15 \mathrm{kHz}$. By using the laser pulse as a trigger for the AD card, a measurement is recorded only when the laser is on. In this way it is simulated that the LDF signal is generated by a cw light source with a power equal to the peak pulse power. Or, in other words: the light source is switched off between two adjacent samples of the AD card. Using a sample-and-hold

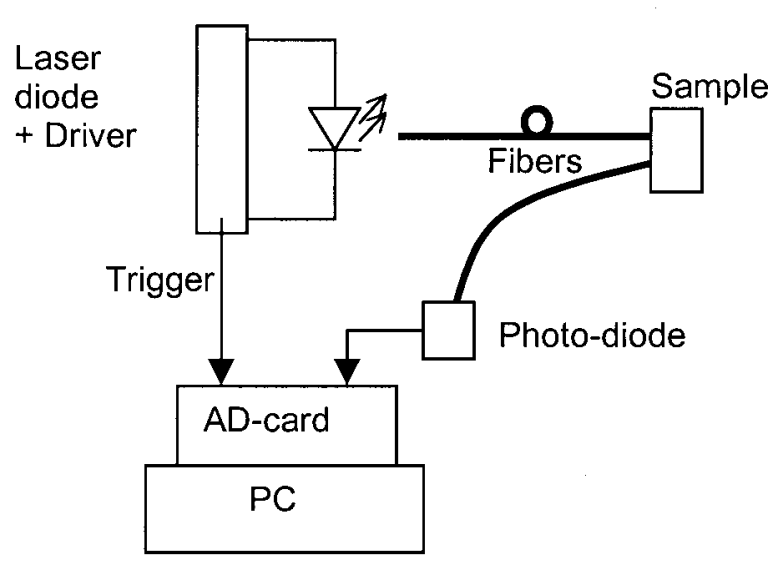

FIG. 2. Pulsed-LDF setup.

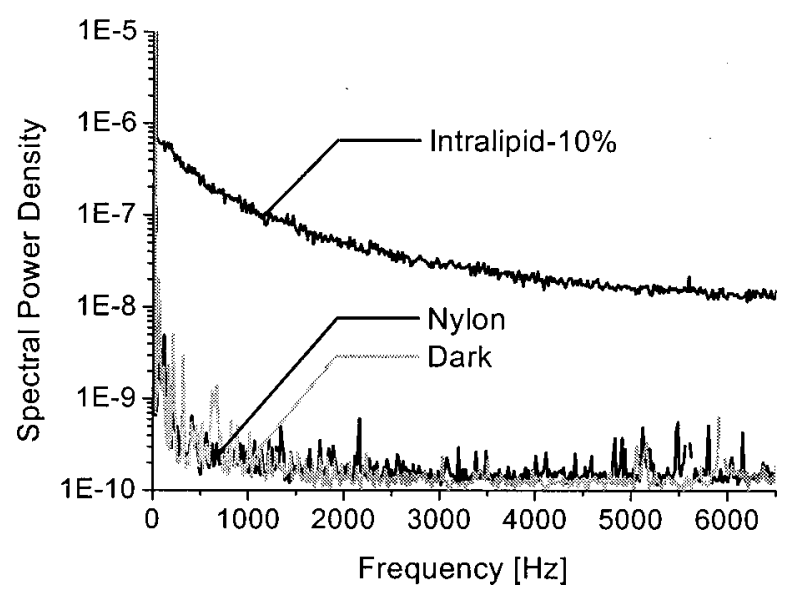

FIG. 3. Pulsed-LDF spectra Intralipid-10\%, nylon, and dark.

circuit, the AD card does not notice that the light source is switched off and measures a power equal to the peak pulse power.

A Labview program is used to collect the signals and to calculate the power spectrum of the detected signals. For each spectrum 1000 data points are collected at a sample rate, determined by the pulse generator $(15 \mathrm{kHz})$. To reduce the noise, several spectra can be averaged. As a sample, an INTRALIPID $^{\text {TM }}$ (IL) suspension is used (IL 10\%). As the fat particles are in Brownian motion ("flow"), the photons will be Doppler shifted. A white nylon block is used as a static ("no-flow") scattering object (no Doppler shifted photons).

\section{RESULTS}

Results of phantom measurements are presented in Figs. 3 and 4 . Figure 3 gives the spectra measured with the pulsedLDF method of a static scattering object (white nylon block, no flow) and a dynamic scattering object (cuvet with IL 10\%, flow, by Brownian motion). The nylon block has similar scattering and absorption properties as the IL 10\%. Also, the spectrum of the background is shown. The spectrum of the IL sample shows the expected Doppler broadening, which is not present in the spectrum of the static scattering object.

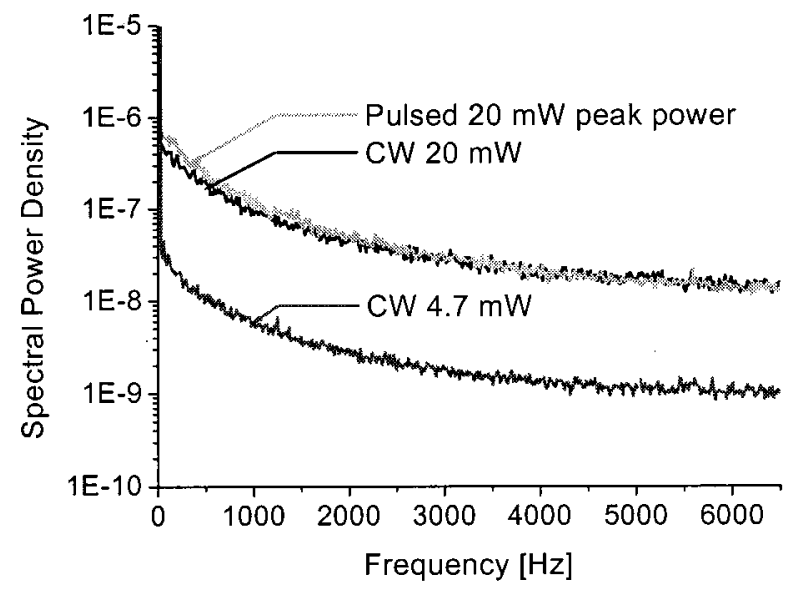

FIG. 4. LDF spectra Intralipid-10\%, cw compared with pulsed LDF. 


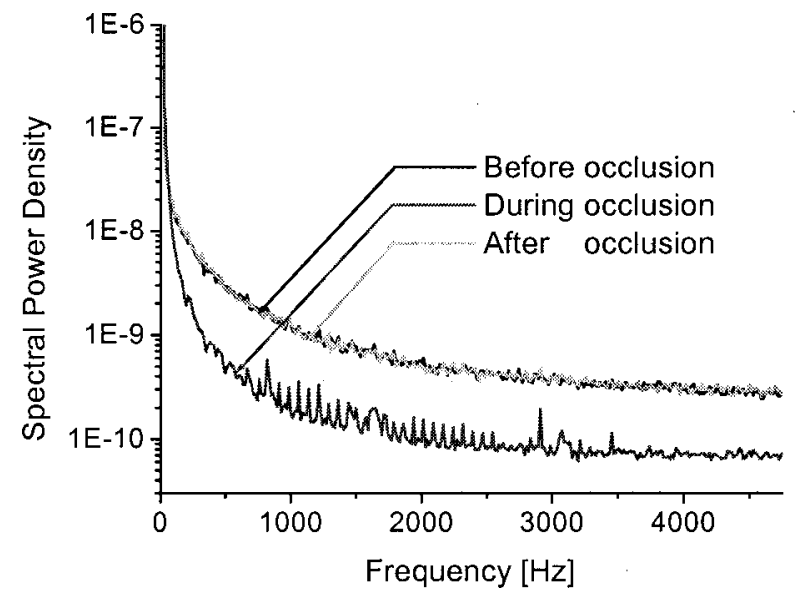

FIG. 5. In vivo, pulsed-LDF spectra of thumb volunteer, before, during, and after occlusion.

Comparison of pulsed and cw LDF has been carried out by comparing the phantom data results of pulsed LDF with a peak pulse power of $20 \mathrm{~mW}$ to $\mathrm{cw}$ LDF with a power equal to the peak pulse power and a power equal to the average pulsed power: $4.7 \mathrm{~mW}$ (Fig. 4). The time ratio of repetition time and length of the pulses was 4.2.

Figure 4 shows that the pulsed-LDF method gives a higher LDF signal than the cw method with the same average power. If the power of the cw LDF method is increased, with a factor equal to the time ratio (4.2), to the peak power of the pulsed-LDF method, both measurements result in the same signal levels.

An in vivo experiment on a thumb of a volunteer, using the pulsed-LDF method, with and without an occlusion has been performed. The spectra are recorded before, during, and after the occlusion.

Figure 5 shows that during the occlusion there are less high frequencies present in the spectrum. Some time after the occlusion, the spectrum is about equal to the spectrum before occlusion.

\section{DISCUSSION}

Figure 3 proves that the pulsed-LDF method indeed measures LDF signals. The LDF spectrum only shows the expected Doppler broadening when a measurement is carried out on a sample with flow (cuvet filled with IL 10\%). This Doppler broadening is not present in the no-flow situation (nylon block).
Furthermore, it is obvious (Fig. 4) that the proposed pulsed-LDF method makes use of the high peak power instead of the lower average power. Figure 4 shows that the pulsed-LDF method gives the same Doppler-signal levels in the spectrum as the cw LDF method with a power equal to the peak pulse power. This proves that the pulsed measurement offers the expected results, using the peak power of the pulses.

Standard cw laser Doppler instruments have a penetration depth up to a few millimeters. This is suitable for monitoring of the microcirculation. To obtain a larger penetration depth, the distance of the fibers must be increased, which is possible when the proposed pulsed-laser Doppler method is used.

Other published pulsed- ${ }^{4}$ or modulated- ${ }^{5}$ LDF methods do not use the high peak power, but use the average power to measure LDF signals. As the peak power can be much higher than the average power, the separation between the source and detector fiber can be increased, resulting in a larger sampling volume, containing information about deep perfusion.

The expected Doppler shifts in tissue cover a frequency range from 0 to $20 \mathrm{kHz}$. This implies that the repetition rate of the pulsed-laser Doppler instrument should be at least 40 $\mathrm{kHz}$ to avoid aliasing. If short pulses $(\sim 50 \mathrm{~ns})$ together with this repetition rate are used, a peak power of 500 times the average power can be achieved.

Possible applications of the proposed pulsed-LDF method include the monitoring of cerebral perfusion as well as monitoring of perfusion through organs (e.g., kidney).

\section{ACKNOWLEDGMENT}

This research was sponsored by the Technology Foundation STW of the Netherlands (Grant No. TTN.4661).

${ }^{1}$ R. F. Bonner and R. Nossal, Appl. Opt. 20, 2097 (1981).

${ }^{2}$ G. Soelkner, G. Mitic, and R. Lohwasser, Appl. Opt. 36, 5647 (1997).

${ }^{3}$ R. Lohwasser and G. Soelkner, Appl. Opt. 38, 2128 (1999).

${ }^{4}$ V. G. Kolinko, F. F. M. de Mul, J. Greve, and A. V. Priezzhev, J. Biomed. Opt. 3, 187 (1998).

${ }^{5}$ H. Olkkonen, Proc. SPIE 1922, 219 (1992).

${ }^{6}$ D. Dopheide, H. Pfeifer, M. Faber, and G. Taux, J. Laser Appl. 1, 40 (1989).

${ }^{7}$ H. W. Jentink, J. A. J. van Beurden, M. A. Helsdingen, F. F. M. de Mul, H. E. Suichies, J. G. Aarnoudse, and J. Greve, J. Phys. E 20, 1281 (1987).

${ }^{8}$ F. F. M. de Mul, J. van Spijker, D. van der Plas, J. Greve, J. G. Aarnoudse, and T. M. Smits, Appl. Opt. 23, 2970 (1984). 